\title{
EL JUEGO ES UN DERECHO Y UNA NECESIDAD DE LA InFfinCIA
}

\author{
Play is for Children Both a heed and a Right
}

JOSÉ LUIS LINAZA

Universidad Autónoma de Madrid

\begin{abstract}
En la introducción de este trabajo se plantea el carácter universal del juego infantil y su relación con las prácticas culturales locales, con referencias a las dos grandes teorías contemporáneas del desarrollo psicológico: Piaget y Vygostky. A continuación se describen los diferentes tipos de juego que se dan en la infancia y la importancia que atribuyeron al juego de ficción los estudios de intervención de la segunda mitad del siglo XX. La relación entre el juego y la cultura, tanto en los juegos de reglas como en los de ficción, muestra la relevancia de esta en la comprensión e interpretación de la actividad lúdica infantil. Finalmente siguiendo el análisis de Bruner sobre la relación entre educación, epistemología y juego, se plantea la necesidad de no reducir la educación a la transmisión de los contenidos del conocimiento, y se aboga por estimular a los niños a explorar el propio proceso de conocer y de comprender, a iniciarse como epistemólogos dirigiendo su atención no solo al producto de conocer sino al proceso de conocer. Sus actitudes en el juego nos deben convencer de que esto es posible.
\end{abstract}

Palabras clave: Juego, Desarrollo, Cultura, Intervención, Epistemología.

Dedicado a Jerry Bruner

\section{Introducción}

Algunos de los más reconocidos psicólogos han señalado la importancia que tiene el juego en el desarrollo humano. Para autores tan relevantes como Freud, Piaget o Vigostky, jugar es una actividad fundamental de nuestro funcionamiento psicológico. Los etólogos han mostrado también su importancia para los organismos inmaduros de otras especies de mamíferos y, muy especialmente, para los primates. En los seres humanos el juego se prolonga a lo largo de toda la vida pero la diversidad de comportamientos que englobamos bajo esta etiqueta de «juego» confunde a muchos de quienes han tratado de estudiarlo y ha llevado a algunos a la conclusión de que se trata de un epifenómeno al que solo el lenguaje otorga entidad propia pero que, al no tratarse de un fenómeno real, no es posible su estudio científico. Tal actitud de negación del juego, y de su posible estudio, responde a una concepción empirista, y un tanto anticuada, del propio conocimiento que, en el ámbito de la psicología, ha defendido 
especialmente el conductismo. Desde otros enfoques, como los de los tres autores mencionados al principio, se busca comprender el complejísimo proceso del desarrollo humano y, para todos ellos, el juego aparece como un relevante factor de dicho desarrollo. Probablemente sigue siendo la teoría piagetiana la que proporciona una explicación más ambiciosa por vincular los diferentes tipos de juegos a los cambios que se producen en las capacidades intelectuales de los seres humanos en el curso de sus vidas individuales. Sin embargo, el objetivo que perseguía Piaget con su teoría era explicar la aparición del pensamiento lógico, que subyace a la producción de la ciencia, entendiendo esta como el mayor logro de la cooperación en la historia de la humanidad. En su reciente libro El mono inmaduro Juan Delval (Delval, 2011) vuelve a proporcionarnos una visión del desarrollo humano como el largo proceso de construcción de las capacidades de razonamiento que caracterizan a los adultos. $\mathrm{Y}$, sin embargo, titula el libro con el rasgo de inmadurez que define a nuestra especie frente a otros primates no humanos. Resulta una cierta paradoja que, al convertirnos en adultos, sigamos conservando rasgos y comportamientos que en otras especies solo definen a los miembros infantiles. Entre tales comportamientos destaca el juego, actividad a la que dedican los niños la mayor parte de su tiempo libre.

Los conceptos de juego e infancia son productos humanos elaborados y modificados a lo largo de la historia. Una infancia prolongada permite adquirir comportamientos complejos, tanto más importantes cuanto más impredecible es el mundo que el futuro adulto debe afrontar. Cuando hablamos de otras especies nos referimos a su «medio natural», al hábitat en el que puede sobrevivir. El medio humano es siempre cultural y solo en interacción con otros podemos sobrevivir y desarrollarnos. Las reglas, y las leyes, constituyen el componente social de dicho medio, tan imprescindible como el medio físico que amplía y modifica la ciencia y la tecnología. Y estas leyes han ido reconociendo como derechos aquellas condiciones y circunstancias que son necesarias para el pleno desarrollo del ser humano. La larga inmadurez de nuestros primeros años de vida impide que podamos interactuar con la realidad, con otros seres humanos, con las capacidades que caracterizan a los individuos adultos de la especie. Afirmar que el juego es una necesidad es reconocer la forma específica con la que los niños abordan la realidad, sea física, social o intelectual. Pero, en la medida en que es necesario para alcanzar nuestra condición humana, se convierte también en un derecho. Los niños tiene derecho al juego como expresión directa de su condición de menores, del mismo modo que no cabe imputarles la plena responsabilidad de sus actos hasta que no hayan disfrutado del tiempo (iy las circunstancias?) necesario en sus vidas para poder desarrollar tal responsabilidad, para hacerse adultos.

Clásicos como Johan Huizinga (1938) plantearon ya, de modo magistral, la relevancia del juego como fenómeno cultural vinculándolo a funciones tan relevantes para el ser humano como el trabajo o el pensamiento. El juego deja de ser una manifestación más de la cultura para convertirse ella misma, en la concepción del célebre holandés, en una manifestación del juego. Por ello, a los títulos Homo sapiens y Homo faber, añade el de Homo Ludens, nombre también de una de sus más reconocidas obras.

Piaget persigue la descripción de universales en el desarrollo infantil. Pero, como no existen niños abstractos, y la psicología se enfrascó durante décadas en una larga y estéril polémica sobre la influencia relativa de la herencia y el ambiente, el tema de las posibles diferencias culturales en el desarrollo humano ha permanecido como banco de pruebas para las teorías evolutivas. Algunos han sostenido que tal desarrollo universal es negado por la evidente influencia de la cultura en la que tiene lugar. Hasta que la teoría de Vygostsky, y el enfoque sociocultural basado en él, no adquirió suficiente reconocimiento en los países occidentales, la polémica 
enfrentó a posiciones conductistas, muy reduccionistas en su concepción del aprendizaje, con el modelo psicogenético, o con el marco psicoanalítico. En las décadas de los sesenta y los setenta del siglo pasado hubo bastantes investigaciones valorando diferentes aspectos del desarrollo infantil en otros contextos culturales y otros continentes y que, en conjunto, reforzaron la descripción piagetiana de grandes estadios en el desarrollo humano.

Quizá, en parte, como resultado de la globalización y de sus efectos sobre las culturas y los individuos, teorías como la de Vygotsky recobran actualidad. Los enfoques sociocultural, ecológico, etnográfico, etc., siguen insistiendo en la relevancia del medio en el que tiene lugar el desarrollo infantil y en la necesidad de su estudio.

En la conceptualización del juego hay grandes coincidencias entre aquellos a los que Bruner (1972a) califica como los tres gigantes del estudio del desarrollo humano: Freud, Piaget y Vygotsky. Pero también hay diferencias, algunas de las cuales pueden confrontarse con datos empíricos. Para los tres, el juego constituye un aspecto central del proceso de desarrollo infantil aunque, cada uno, ponga el énfasis en funciones diferentes. Para Vygotsky, teorizando fundamentalmente desde el juego de ficción o juego sociodramático, este es un factor que lidera el desarrollo, un ejemplo de su Zona de Desarrollo Próximo, puesto que el niño realiza en el ámbito lúdico muchas actividades, fingidas, que aún no puede realizar en el mundo real. Freud vincula directamente el juego con la realización de deseos inconscientes, como los sueños para los adultos, y le asigna un papel relevante en el diagnóstico y en la terapia. Piaget vincula los tipos de juegos a las grandes estructuras de conocimiento (esquema sensorio-motriz, símbolo y operación mental) y les atribuye funciones diferentes en función de ello. El juego de ejercicio explora y consolida habilidades muy diversas a lo largo de la vida. El juego de ficción amplía el mundo real, con el que interacciona el niño, a mundos fantásticos del pasado, presente o futuro. Los juegos de reglas establecen las primeras sociedades infantiles en las que poder actuar juntos y establecer límites y reglas con los que enfrentar los conflictos.

\section{Los primeros juegos infantiles}

No todos los diferentes tipos de juego están presentes en los seres humanos en cualquier momento de la vida. Los primeros juegos van apareciendo con el control que el niño logra de su propio cuerpo: agarrar, chupar, golpear, etc. Son los juegos funcionales o juegos motores, propios de los dos primeros años de vida, y en los que se ejercita cada una de las nuevas habilidades adquiridas. Soltar y recuperar el chupete constituirá un juego típico de un niño de pocos meses, mientras que abrir y cerrar una puerta, o subir y bajar escaleras, aparecerán más tarde.

Entre los primeros objetos por los que se interesa el bebé ocupa un lugar destacado la madre que, además, se comporta de modo muy diferente a cómo reaccionan los objetos físicos. Estos juegos de interacción social (dar palmas, identificar y nombrar los dedos de una mano, esconderse y reaparecer, etc.) también experimentan una curiosa evolución. En sus comienzos el niño los sufre pasivamente. Es el adulto quien le lleva las manos, le oculta, le hace reaparecer. En pocos meses el pequeño invertirá los papeles, tomando la iniciativa, y será él quien identifique y nombre los dedos del adulto o quien pretenda sorprenderle con su reaparición súbita en un juego de cú-cú-tras-tras. El cambio de papel es posible porque el niño capta la estructura de la interacción en la que ambos jugadores desempeñan actividades complementarias.

Con objetos o con personas, el juego discurre en el presente, en lo que podríamos definir como el aquí y ahora. Pero solo los humanos construyen objetos que estimulen y faciliten 
esas primeras exploraciones del mundo físico: chupetes, sonajeros, móviles, etc., así como instrumentos cotidianos, especialmente adaptados a sus cuerpos pequeños y, todavía, poco hábiles (sillas, cubiertos, andadores, etc.).

Con la aparición de los juegos de ficción se produce un cambio profundo en la actividad infantil. Objetos y gestos se transforman para simbolizar otros que no están presentes —una muñeca que representa una niña, un palo que hace de caballo, un dedo extendido se pretende pistola, etc.-. Lo fundamental no son las acciones sobre los objetos, sino lo que estos y aquellas representan.

Buena parte de estos primeros juegos de ficción son individuales o, si se realizan en presencia de otros niños, equivalen a lo que se ha llamado juego en paralelo, en el que cada jugador desarrolla su propia ficción con esporádicas alusiones al compañero para informarle o pedirle que ratifique aspectos muy generales del mismo. A pesar de ello, este juego que Piaget denominó egocéntrico no siempre se caracteriza por la impermeabilidad a las acciones y sugerencias de los demás compañeros de juego. El análisis de las conversaciones infantiles mientras juegan muestra que, desde los tres años, los niños diferencian las actividades que son juego de las que no lo son y que, cuando la situación es ambigua, recurren al lenguaje para hacérsela explícita unos a otros (Garvey, 1977).

Esta simultánea representación de ficción y realidad es una condición del juego simbólico, pero no su objetivo. La meta de estos juegos, de todo juego, es la acción, actuar, no imitar. Algunos de estos juegos son una especie de resumen o versión simplificada de la realidad social, observada o experimentada, por los propios niños. Jugar a las casitas, a las tiendas, a los colegios, o a cualquier otro tema de la vida cotidiana de los adultos familiares, solo exige de los jugadores una actualización de esos modelos, una especie de evocación en voz alta de los roles y las interacciones entre ellos, similares a las que se producen en las situaciones de la vida real.

Por el contrario, jugar a temas poco familiares o fantásticos, como guerras medievales o espaciales, supone establecer entre los jugadores un mundo de ficción compartido que tiene poco que ver con la experiencia directa de los propios jugadores. Estos juegos de ficción compartidos interesaron especialmente a Vygotsky (1979) y a sus discípulos y, en la década de los ochenta, se divulga en Occidente su concepción del juego infantil, desarrollada posteriormente por Elkonin (1980), Bruner (1984, 1988), Rogoff y Werstch (1984), etc. Vygotski interpreta los juegos sociodramáticos como una forma de ensayar conductas del mundo adulto, atribuyendo a los objetos un significado personal y cultural. También Bruner (2002) destaca el papel del juego como mecanismo de transmisión cultural, apoyándose en los planteamientos de Piaget y Vygotski y situando al niño como un miembro activo más de la cultura en la que se desarrolla. Por otra parte, esta subcultura de los juegos infantiles ha interesado a estudiosos e investigadores que sentían la necesidad de recogerlos, junto con canciones, cuentos, etc., ante el miedo a su probable desaparición (Opie y Opie, 1969).

\section{El juego simbólico y los estudios de intervención}

Es cada vez más frecuente la crítica a las investigaciones sobre el juego infantil, y a sus posibles aplicaciones, denunciando que su valoración proceda exclusivamente por su contribución a otros aspectos del desarrollo (cognitivo, social, lingüístico, afectivo, etc.) y no se plantee la relevancia del juego por sí mismo, como característica de la infancia (Karnik y Tudge, 2010), y hasta como un derecho de reciente reconocimiento.

Efectivamente, la mayoría de las investigaciones de las décadas de los sesenta y setenta del siglo pasado planteaban el posible beneficio 
del juego en otros ámbitos del desarrollo. Por ejemplo, en el desarrollo cognitivo (Piaget, 1946), en el desarrollo afectivo (Erickson, 1972), en la resolución de problemas (Bruner, 1972b; Sylva, Bruner y Genova, 1976), en la capacidad de categorizar la experiencia (Bateson, 1955), en el desarrollo social (Parten, 1932; Linaza y Maldonado, 1987), en la adquisición del lenguaje (Vygotsky, 1978; Garvey, 1990; Nelson, 2006), etc. En su revisión de la literatura sobre juego, Rubin, Fein y Vandenberg (1983) comentan muchas investigaciones que han tratado de establecer relaciones entre el desarrollo del juego y el incremento de las capacidades lingüísticas, de resolución de problemas, de capacidad para ponerse en el lugar de otro, de creatividad, etc.

En esta lógica de la positiva influencia del juego en el desarrollo infantil, aparece la posibilidad de que la ausencia o la escasez de juego tenga una incidencia negativa en ese desarrollo. A partir de ahí se produce un conjunto de circunstancias que influyeron mucho en lo que se han llamado estudios de intervención. Por ejemplo, para valorar la cantidad y calidad de los juegos de ficción no eran los laboratorios de los departamentos universitarios el mejor contexto para generarlos. Tampoco los laboratorios móviles o rodantes, que se instalaban cerca de las escuelas para pedir a los niños que entraran a jugar en ellos. Y, una vez confirmada en estas condiciones la escasa actividad lúdica, se plantea la intervención psicológica, experimental, para compensar los déficits, observados o supuestos. Por ejemplo, varios estudios de esos años (Smilansky, 1968; Eifermann, 1970; Fein, 1981, etc.) plantean la intervención educativa para mejorar el nivel de juego, o para compensar los déficits derivados de su escasa presencia en niños (los déficits aparecían en los juegos de niños árabes comparados con los israelíes).

Cuando los adultos, al reconocer la influencia positiva del juego en el desarrollo, deciden intervenir y controlar el juego, transforman este en otra actividad diferente, impuesta y controlada por ellos. Los niños pierden, en estos casos, su protagonismo y el juego deja de ser tal. Los trabajos de intervención son, desgraciadamente, ejemplos de confusión entre lo que denomina juego el adulto (experimentador, educador o padre) y lo que el niño decide que es juego. Ni todos los objetos ni todas las situaciones son igualmente eficaces en provocar, sostener o estimular el juego.

Entendemos que los juegos son al mismo tiempo instituciones sociales y sistemas de relaciones personales. Los guiones aluden a regulaciones sociales que trascienden las interacciones personales y las reglas son convenciones que estructuran los grupos humanos (Linaza y Maldonado, 1987). En nuestros estudios sobre el juego de ficción nos apoyamos en dos tradiciones diferentes. Por una parte, aquella que analiza las prácticas infantiles como la construcción conjunta de un guión. Tomando la idea de Schank y Abelson (1977) sobre la organización de la información en la memoria de sujetos adultos, entienden el juego de ficción como un guión compuesto de una secuencia de actos, organizados alrededor de unas metas, y especificando roles de actor, objetos y escena (Nelson, 1978, 1981; Nelson y Gruendel, 1979). Según Nelson y Seidman (1984), el juego de ficción compartido se puede definir como un diálogo organizado alrededor de un contexto común de ficción, y este contexto se crea al aportar los niños alguna representación de la realidad, que se establecerá en la ficción. Cuando se alude al contexto de estos guiones se está refiriendo tanto a aquel que describe la representación que tienen los niños sobre la realidad representada, como al contexto físico y social del propio juego (Nelson y Seidman, 1989). En otros trabajos el contexto señalado es el conversacional, aquel que contribuye al mantenimiento del diálogo entre los jugadores. En este caso son tres sus componentes: la presencia de objetos, el tema compartido y el conocimiento del guión que se comparte (Nelson y Gruendel, 1979). Resulta muy útil el concepto de guiones compartidos, ya que cuando un guión se convierte en tema de 
juego proporciona la estructura dentro de la cual puede desarrollarse una secuencia coherente de conversación y de actividad lúdica.

La segunda tradición es la que compara el juego en diferentes culturas. En el caso de un juego como el palín, practicado por los mapuches en Chile, su comprensión y análisis requiere entenderlo como práctica cultural de esa sociedad y que no es, ni un juego de niños, ni una actividad deportiva tal y como la entendemos en nuestra cultura occidental (Garoz y Linaza, 2006).

Al estudiar prácticas de crianza en diferentes culturas, Rogoff y col. (1993) mostraron que las interacciones entre cuidador y niño varían de una cultura otra. Compararon cuatro contextos de crianza diferente: dos, urbanos y de clase media, de Estados Unidos y Turquía (Salt City, Kecioren); y dos, de clase baja y más agrícolas, de Guatemala e India (San Pedro y DholKi-Patti). Evidentemente son diversas las dimensiones en las que difieren entre sí estas cuatro comunidades y, por ello, los investigadores optaron por aceptar como unidad de comparación a cada una de ellas, y no variables individuales como clase social, nivel de educación de los padres, rural o urbano, etc. Encontraron que había diferencias notables y que, por ejemplo, la integración de los niños en las actividades diarias de los adultos era mayor en las comunidades de EE. UU. y Turquía, que en las de economía de subsistencia de Guatemala e India.

Esta comparación entre culturas en el ámbito del juego (Göncü, Mistry y Mosier, 2000; Göncü, 2011) muestra que este varía en función de a quién consideran los adultos de cada comunidad compañeros adecuados de juegos. Del mismo modo, la posibilidad de interactuar con adultos, familiares o no familiares, influye en el tipo y tamaño del grupo de juegos. Respecto a la preocupación que generaron los experimentos de intervención, el hecho de que no se practique un tipo de juego concreto no significa que a los niños se les prive del beneficio concreto que ese juego pueda proporcionar. Se puede obtener ese beneficio participando en otros juegos, o a través de una actividad no lúdica. La conclusión de estos estudios indica que los tipos de juegos, en los que participan los niños, dependen de lo que las comunidades consideran como adecuado para jugar. Es decir:

«.. el juego de los niños refleja las creencias de los adultos sobre el desarrollo y la estructura social en la que los niños crecen» (Göncü, 2011).

En parte por la misma divulgación y popularización de los conocimientos psicológicos, las familias de clase media, americanas, europeas o turcas valoran el juego de ficción. En zonas campesinas de América Latina, África o la India, los niños se incorporan a las actividades de los adultos y a las tareas domésticas, como cuidar de sus hermanos pequeños, de los animales, lavar, coser, cocinar, etc., y la valoración del juego es diferente y, en algunos estudios, menos frecuente y con menor participación de los adultos.

También cabe que se produzcan ambas actividades simultáneamente. En Madagascar, por ejemplo, las niñas que observamos jugaban a las mamás con sus hermanos pequeños, de quienes se estaban ocupando mientras las madres cocinaban, fingiendo que les daban de comer o que les acostaban para dormir. Y tales conductas se producían tanto en la capital, Antananarivo, como en poblaciones rurales. De modo que, mientras trabajan o cuidan de sus hermanos, también juegan.

Se puede decir que en las últimas décadas ha habido un renovado y creciente interés por los contextos socioculturales del juego. Cabe pensar que, frente a lo que sucedía en la década de los sesenta del siglo pasado, con una proyección de los estudios de niños blancos de clases medias hacia el desarrollo en contextos no occidentales, hoy se produce un mayor 
contacto de unas culturas con otras, hay un incremento de los desplazamientos de poblaciones enteras a otros países y, por tanto, un aumento de la comparación entre prácticas culturales diferentes. Entre ellas, el cuidado y la educación de niños pequeños y el modo de concebir lo que es apropiado o inapropiado para la infancia.

\section{Juego y desarrollo}

El trabajo pionero de Piaget sobre el desarrollo humano sigue siendo una referencia fundamental a pesar del tiempo transcurrido. Sus propuestas de estructuras de conocimiento (esquemas sensorio-motores, operaciones mentales concretas y operaciones mentales formales) pretendían describir características universales del desarrollo de los seres humanos, en buena medida independientes del contexto cultural o del momento histórico en el que tenga lugar dicho desarrollo. También su explicación del desarrollo social y moral (Piaget, 1932) sigue inspirando numerosas investigaciones. Sin embargo, la concepción individualista del funcionamiento psicológico, compartida por la mayor parte de los enfoques teóricos contemporáneos, ha dificultado la incorporación y el estudio de las interacciones sociales como un factor de desarrollo y como un mecanismo constituyente de la psique humana. Y así, la práctica de los juegos de reglas, y el desarrollo del conocimiento social que genera, ocupa en la teoría piagetiana, un papel central en el desarrollo infantil. Y, como en el caso de las estructuras cognitivas, se ha supuesto que las etapas de este desarrollo socio-moral serían también universales. Pero, así como el mecanismo de desarrollo de las estructuras cognitivas es la actividad individual del sujeto, en el desarrollo social el propio Piaget reconoce, como mecanismo de desarrollo, las interacciones entre iguales. La dicotomía entre relaciones heterónomas-autónomas llevó a Piaget a estudiar los juegos tradicionales como el ámbito privilegiado en el que se regulan y desarrollan las relaciones entre iguales y la autonomía moral.
A partir de estos estudios, Linaza y Maldonado (1987) estudiaron cómo adquieren los niños las reglas del fútbol. Era, y sigue siendo, el juego más popular para la mayoría de los varones y para un número creciente de chicas. Pero, a diferencia de otros juegos tradicionales estudiados por Piaget (1932) y por otros autores (Linaza, 1984), el fútbol es también, en tanto que deporte, una práctica adulta. Los resultados obtenidos sobre la conciencia de las reglas del fútbol muestran que, los conflictos y negociaciones entre iguales, dan lugar a la comprensión del acuerdo mayoritario como mecanismo de regulación, legitimación y cambio de las reglas, ratificando los descubrimientos piagetianos. Sin embargo, los sujetos mayores son muy sensibles a las regulaciones del deporte que sobrepasan las meras prácticas infantiles e invocan la necesidad de acuerdos «de mucha gente», «de las federaciones», para poder establecer y legitimar cambios en las reglas (Linaza y Maldonado, 1987: 145-148). Aunque los autores no lo formularan explícitamente en el estudio original, las argumentaciones de estos niños y adolescentes remiten al significado de los deportes en nuestras sociedades actuales, desbordando la mera práctica lúdica infantil, y situando los juegos y los deportes como elementos de las culturas en las que se aprenden y transmiten.

El estudio de los juegos tradicionales en sociedades indígenas nos proporciona una perspectiva novedosa sobre los mecanismos de regulación y legitimación de normas sociales. El análisis de la adquisición del conocimiento práctico de las reglas, las acciones e interacciones que los definen, no mostró grandes discrepancias respecto a la adquisición de las mismas en otros juegos tradicionales y en otros deportes (Garoz, 2005, Garoz y Linaza, 2006). Sin embargo, el conocimiento teórico, o la conciencia de las reglas, muestra una clara opción de los niños y adolescentes mapuches por las reglas tradicionales. No hay nada que impida poder modificarlas excepto la voluntad de los propios mapuches por conservar una práctica 
que forma parte de su identidad como pueblo (Garoz y Linaza, 2006: 41-42). Estas referencias a valores y regulaciones, que van más allá de lo que sucede en el terreno de juego, explican el interés de los autores por el significado del Palín, su relación con otros aspectos de la cultura mapuche, y por los posibles cambios que tienen lugar en el proceso de construcción del conocimiento sobre dicho juego.

Piaget reconoce la importancia de las interacciones sociales como uno de los factores de desarrollo, pero dedicó la mayor parte de su esfuerzo a poner de manifiesto la universalidad del factor de equilibración y su aportación específica al desarrollo individual. Pero es evidente que tal desarrollo no se produce en el vacío y que, como señala Delval (1997), es en parte un diálogo con lo que rodea al niño, una interacción con los demás miembros de la sociedad bajo unas determinadas regulaciones sociales, donde los adultos moldean las ideas de los niños y transmiten las ideas dominantes de la sociedad. Cuando hablamos de «sociedades tradicionales», es frecuente suponer que esta presión de los mayores es más explícita en ellas y que constituye una de las causas para que los cambios culturales y generacionales sean menores.

\section{Juego y cultura}

Complementaria a esta visión sobre el desarrollo del juego es la que aporta la perspectiva sociocultural o sociohistórica. Los trabajos de Vygotsky o Wallon, plantean la necesidad de analizar las relaciones existentes entre el contexto sociocultural y el desarrollo humano. Dentro de esta perspectiva tanto Vygotski (1979), como Elkonin (1980), abordan el estudio del juego dentro de una visión más histórica y contextualizada del desarrollo humano. Conciben el juego como una reconstrucción de las interacciones de los adultos, que el niño entiende solo de un modo fraccionado, y que solo puede tener lugar gracias a la cooperación e interacción social con otros niños que asumen papeles complementarios del suyo. Por esta misma razón, cuando la teoría aborda el caso de los juegos de reglas, los define como un mero cambio en el énfasis de sus componentes de ficción y de regulación. Probablemente por esa equiparación de los juegos de reglas a los llamados sociodramáticos, la influencia de estos autores ha sido mayor en los estudios sobre los juegos de ficción, en los que el contenido de los guiones del juego reflejan características de las relaciones adultas en cada cultura, que en los de juegos de reglas. Sin embargo, la formulación explicita de la reglas transforma cualitativamente los juegos y la regulación de conflictos y acuerdos. La regla anticipa y prescribe el acuerdo pero, también, permite jugar con la regla, actuar con ella y reflexionar sobre ella. Adquiere el status de realidad propia, se objetiva. Y este aspecto es especialmente relevante respecto a la relación entre la educación, el juego y la epistemología que señala Bruner (2012) y al que nos referiremos en la última parte de este artículo. Pero, para entender la complejidad de esta relación entre juego y desarrollo también son muy relevantes otros de sus trabajos elaborados a lo largo de décadas (Bruner 1972, 1991, 1996, 2005). Aunque la cultura sea un producto de la acción y la mente humanas, la propia mente es también producto de las culturas concretas en las que se desarrolla. Respecto al papel del juego en el desarrollo, Bruner entiende que en la infancia el juego es un instrumento de socialización y de transmisión cultural, ya que la incorporación a la cultura humana y a las destrezas adquiridas a lo largo de las sucesivas generaciones no se puede realizar solamente desde la experiencia individual y directa.

Cabe interpretar que, en los planteamientos de Piaget, Vygotski o Bruner, el niño no es solo un ser que desarrolla su inteligencia mediante el juego, o que incorpora el mundo de los adultos a través de él, sino que se le otorga un papel de miembro activo en la cultura en la que participa, en la que realiza sus potencialidades y a través de la cual adquiere toda una serie de significados 
públicos y compartidos (mediante procedimientos de interpretación y negociación).

Respecto a la relación entre un tipo de juego y la cultura de referencia, deben mencionarse los estudios de Elkonin (1980), para quien la variedad de juegos existentes depende tanto de las épocas históricas como de las condiciones sociales, geográficas o culturales. Y, también, los estudios de Roopnarine, Johnson y Hooper (1994), en los que a través de la observación de juegos de la India, Taiwán, África y de la población esquimal, plantean que cada juego mantiene una estrecha relación con unos valores, tradiciones, rituales o religiosidad determinados, y es el resultado de participar en un ambiente cultural o subcultural específico. Para otros autores se trata, además, de actividades cambiantes por la evolución de las costumbres familiares y los avances tecnológicos (SuttonSmith y Roberts, 1964, 1981; Bathiche y Derevensky, 1994). Por eso insisten en la importancia de estudiar los juegos teniendo en cuenta su papel y relevancia específicas, su significado y trascendencia. Ignorar el contexto sociocultural, las variantes que cada cultura aporta al desarrollo individual, no ayuda a comprender el papel del juego en este (Roopnarine, Jonson y Hooper, 1994; Pellegrini, 1995). Se sabe de la importancia que tienen algunos juegos motores en la adquisición de habilidades físicas especialmente valoradas en las culturas que los practican y fomentan. También son conocidas algunas relaciones entre los guiones de los juegos de ficción y las prácticas sociales adultas en las que se inspiran. Pero se sabe menos sobre las teorías implícitas (conciencia de la regla) que elaboran los niños de diferentes culturas respecto a los mecanismos de regulación, negociación y legitimación de dichas reglas.

Como señalan Linaza y Maldonado (1987), es solo a partir del siglo XIX cuando en las sociedades industriales se crean las prácticas deportivas, adquiriendo unas funciones y significados que no tenían los juegos tradicionales. Es decir, en nuestras sociedades occidentales podemos observar el estrecho vínculo que existe entre los juegos de reglas y los deportes, estableciéndose entre ellos, en algunas ocasiones, una relación de continuidad. Tan importante es esa relación que, en ocasiones, resulta difícil clasificar una actividad como deporte o simplemente como juego. Pero es un factor que puede influir en el tipo de interacción que define estas actividades, y en la consiguiente conciencia de la regla. El propio Piaget sugería que si el juego de las canicas se prolongara hasta los 18 años, los niños llegarían más tarde a la conciencia de la autonomía que en el caso de los estudiados por él, ya que la conciencia democrática solo es posible por la práctica de la cooperación entre iguales. Y es que los deportes prolongan prácticas lúdicas infantiles pero, al mismo tiempo, en tanto que actividades adultas reguladas, institucionalizadas y competitivas, incorporan una presión externa que no tenían los juegos.

Cabe plantearse si los valores del deporte, elaborados en contextos históricos y sociales concretos, serán igualmente válidos para todas las culturas del mundo. La diversidad y variedad actuales, tanto en el juego de los niños como en el de adultos, en distintas regiones del mundo, es más amplia que la de los deportes de la tradición europea y americana. En muchos lugares, incluidos los poblados mapuches, deportes como el fútbol son practicados por los niños indígenas tanto como sus juegos tradicionales.

Entender cómo construyen los significados de estas actividades es tan relevante para nuestra comprensión del desarrollo humano como lo es entender la adquisición de las reglas prácticas o los procedimientos por los que se elaboran, legitiman y modifican sus reglas.

\section{Metodologías, datos empíricos e interpretación de los juegos}

Es importante insistir en una característica del juego que explica la dificultad de realizar estudios experimentales sobre él. El juego es una 
actitud ante la realidad, un modo de actuar, que define el propio jugador y no puede ser impuesto desde fuera. Algunos estudios sobre el juego infantil, tratando de garantizar un diseño experimental, terminan investigando comportamientos y actuaciones sobre objetos que los investigadores definen como juguetes pero que no son juego.

También es muy relevante quién hace las observaciones y cómo afecta la propia observación al juego. Se necesitan tiempo y empatía para establecer las condiciones que permitan realizarlas. Por ejemplo, en el caso del juego mapuche del palín, Garoz (Garoz y Linaza, 2006) dedicó semanas a participar en sus juegos y, como jugador de hockey, logró sorprender a los propios mapuches por las habilidades de un winka (extranjero) con el palo. El valor de sus observaciones participantes y de sus entrevistas clínicas no es independiente del hecho de haber sido aceptado como jugador. Como en otros ámbitos del desarrollo infantil, en la literatura sobre juego hay muchos estudios en los que las acciones y las interacciones responden a situaciones creadas por el investigador. Se priman observaciones en contextos artificiales para los niños: laboratorios, visitas de adultos extraños al hogar, instrucciones y objetos específicos, etc. La dificultad es enorme para poder observar directamente el desarrollo infantil y, si cabe aún más, el juego de ficción espontáneo. Porque niños y niñas elaboran estos juegos en espacios y tiempos libres del control adulto. Quien recoge los datos realiza las observaciones y las entrevistas, es un punto de enorme relevancia. En las ciencia naturales tiene una enorme importancia el potencial y la precisión de los aparatos utilizados. En el caso del juego infantil los procedimientos, las observaciones naturales, las entrevistas condicionan igualmente el valor y la relevancia de los datos obtenidos.

En las últimas décadas la psicología cultural, los enfoques sociocultural y ecológico, las metodologías longitudinales, la influencia de otras disciplinas sociales, como la antropología y la etnografía, o la sociología y la sociolingüística, han contribuido a modificar profundamente la concepción de la influencia de la cultura en el desarrollo infantil (Bruner, 1996). Ha dejado de ser una variable a controlar en los diseños para concebirse como una parte integral del funcionamiento de cualquier ser humano.

Los contrastes entre culturas occidentales frente a no-occidentales han ido dejando paso a una aceptación de la heterogeneidad dentro de cada cultura. Y a comprobar, por ejemplo, que puede ser más parecido el desarrollo de un niño en un medio urbano, aunque sus ciudades pertenezcan a continentes diferentes, que entre esos mismos niños y sus iguales en zonas rurales del mismo país. O entre dos niños de clase media, de diferentes países, que entre estos y dos niños de clase obrera. Hay culturas diferentes dentro de una misma sociedad, país o nación (Göncü y Abel, 2009).

En un trabajo reciente (Linaza y Bruner, 2012) nos hemos referido a tres ejemplos concretos de estudios sobre el desarrollo del juego infantil en tres contextos socioculturales muy distintos y en tres países diferentes: España, Chile y Libia. Sin embargo, cada uno de ellos es un buen ejemplo de la diversidad de culturas en el interior de cada país. También son un excelente ejemplo de la importancia que tiene quién y cómo obtiene los datos del estudio. En el caso de los niños pasiegos, en la Vega de Pas, Ofelia García y Beatriz Martín Andrade dedicaron muchos meses y mucha habilidad social para llegar hasta los juegos de vacas y de muñecas que observaron (García, Martín-Andrade y Linaza, 1996). Las observaciones de los juegos de las Visitas mapuches, realizadas por Nilsa Rain, muestran que la autora reúne cuatro roles difíciles de aunar en una misma persona: madre, maestra, dirigente cultural e investigadora (Rain, 2011). Y esa triple condición de madre, maestra e investigadora está presenta también en Massauda, en su estudio del juego de los niños libios (2012). 
La relación del juego con lo que sucede en la sociedad adulta es muy evidente en estos tres estudios. Los juegos de las vacas pasiegos aludían a la actividad cotidiana de padres y madres en cada familia pasiega. Por ejemplo, aparecían en los juegos la preocupación a una posible enfermedad de las vacas por «haberse tragado un clavo» mientras pastaban. En el caso de los juegos mapuches aparecen reflejados los conflictos sociales por las reivindicaciones de sus territorios ancestrales, y la consiguiente represión por parte de las fuerzas policiales, los carabineros. Existe incluso un juego con ese título: mapuches contra carabineros. Pero la conciencia de la fragilidad de los mapuches, frente a las cargas y detenciones de los carabineros, ha provocado que la mayoría de los niños mapuches no quieran jugar a ser mapuches, sino carabineros (Rain, 2011). La influencia en la elección de los roles no está restringida a las experiencias directas sino que se ve influenciada también por las indirectas. En un juego muy popular en la España de mitad del siglo XX, indios y vaqueros, los roles no estaban inspirados en experiencias directas de los niños sino en las numerosas películas del Lejano Oeste, épica de los colonizadores vaqueros en lo que llamaron La Conquista del Oeste y que fue, en realidad, la ocupación violenta de las tierras ocupadas por los pueblos originarios de América. En el caso más reciente de la guerra civil en Libia, los niños juegan a los soldados de Gadafi frente a los rebeldes (Ahmed, 2012). De nuevo, el juego infantil alude a los conflictos que se dirimen en la sociedad adulta.

\section{Juego, educación y epistemología}

Hemos insistido en la importancia de las diversas adquisiciones sociales, cognitivas, lingüísticas, motrices, etc., que niños y niñas logran en la práctica de sus juegos. Conocerlos, observarlos, fomentarlos es responsabilidad de los adultos que les rodean. Proporcionarles espacios y oportunidades para jugar es la mejor manera de atender a su derecho a jugar. Niños y niñas necesitan jugar para vivir con plenitud su infancia. Finalizaremos con una reflexión sobre la compleja relación entre juego, educación y epistemología a la que aludíamos anteriormente. El trabajo de Piaget (1932) constituye una referencia clásica a la toma de conciencia de las reglas a partir de la cual otros autores hemos podido constatar la relevancia de esta toma de conciencia en la práctica social del juego, por ejemplo, para poder utilizar la regla como estrategia (Linaza y Maldonado, 1987), y en la comprensión del origen y la naturaleza de las propias reglas sociales (Garoz y Linaza, 2006). En un reciente artículo, Bruner enriquece y amplía esta perspectiva epistemológica sobre nuestra construcción del conocimiento y la asocia directamente con la propia educación infantil. Y, así, señala:

«... hemos aprendido mucho sobre el modo en que nuestra especie se reinventa a sí misma para afrontar tanto las limitaciones de nuestra naturaleza biológica como las oportunidades de los mundos culturales que creamos. Y esto tiene profundas consecuencias para la educación.

La educación no es solo, ni debe dedicarse exclusivamente, a la transmisión del conocimiento establecido. Se debe dedicar también a cultivar la conciencia de la condición humana y a generar la habilidad de comprender la naturaleza y las fuentes del conocimiento. Es decir, la educación no consiste solo en llegar a dominar un contenido sino que también consiste en lograr captar en qué consiste conocer y comprender. Sí, estoy afirmando que deberíamos cultivar en nuestros escolares una sensibilidad epistemológica apropiada, una conciencia de los procesos implícitos en aprender y en pensar y no únicamente en los productos finales de dichos procesos a los que llamamos curriculum. Es absurdo decir que los niños no son capaces de comprender estos procesos. Sus actividades de juegos espontáneos están llenas de exploraciones sobre lo posible, sobre lo que podría ser y sobre por qué en ocasiones es posible y en otras no. 
Considero de extremada urgencia que cultivemos este sentido de lo posible en nuestras prácticas educativas» (Bruner, 2012: 13).

Es una propuesta ambiciosa, tanto para los educadores como para quienes estudian el desarrollo infantil. Es, además, original y lúcido utilizar el juego como banco de pruebas para esta tarea. Permítaseme finalizar agradeciendo la iniciativa a su autor y felicitándole en su 97 aniversario con la dedicatoria de este artículo.

\section{Referencias bibliográficas}

Ahmed, M. (2011). El juego en el desarrollo psicológico de los niños libios. Tesis doctoral en preparación, Universidad Autónoma de Madrid.

Bateson, G. (1955). A theory of play and fantasy. Psychiatric Research Reports, 2, 39-51.

BRUner, J. S. (1972a). Concepciones de la infancia: Freud, Piaget y Vygotsky. En J. LinAzA (comp.) Acción, pensamiento y lenguaje: Escritos de J. S. Bruner (31-44). Madrid: Alianza (1984).

Bruner, J. S. (1972b). Inmaturity: its nature and uses. American Psychologist 27, 687-708. Trad. española en J. LinazA (comp.), Acción, pensamiento y lenguaje: Escritos de J. S. Bruner, pp. 45-. Madrid: Alianza (1984).

Bruner, J. S. Acts of meaning (1990). Cambridge, M. A.: Ed. Harvard University. Trad. Castellano en Madrid: Ed. Alianza (1991).

Bruner, J. S. (1996). The Culture of Mind. Cambridge, M. A.: Ed., Harvard University Press. Trad. castellano La cultura puerta de la educación. Madrid: Visor (1997).

BRUNER, J.S. (2012). What Psychology Should Study. International Journal of Educational Psychology, 1 (1), 5-13.

Delval, J. (2011). El mono inmaduro. Madrid: La Catarata.

- (1997). El conocimiento social. En J. A. García Madruga \& P. Pardo (eds.), Psicología Evolutiva, tomo 2 (pp. 85-115). Madrid: UNED.

EIfERmanN, R. (1970). Level of Children's Play as expressed in group size. British Journal of Educational Psychology, 40 (2), 161-170.

Elbers, E. (1994). Sociogenesis and children's pretend play: A variation on vygotskian themes. En W. De GraAf y R. MAier (eds.), Sociogenesis reexamined. Nueva York: Springer.

Elkonin, D. B. (1980). Psicología del juego. Madrid: Pablo del Río.

ERICKSON, E. (1972). Play and Development. Nueva York: Norton

FEIN, G. (1981). Pretend play in Childhood: an integrative view. En Child Development, 52, 10951118.

García, O., Martín-Andrade, B. y Linaza, J. (1996). El juego simbólico en los niños pasiegos: muñecas y vacas. Revista cultura y educación, 1, pp. 77-85.

GAROz, I. (2005). Juegos y deportes. Un estudio del palín mapuche y el hockey hierba. Madrid: UAM Ediciones.

Garoz, I. y LinazA, J. (2006). Juego, Cultura y Desarrollo en la Infancia:el caso del Palín Mapuche y el Hockey. En Revista Internacional de Ciencias del Deporte, 2, 33-48.

Garvey, C. (1977). Play. En J. Bruner, M. Cole y B. Lloyd (eds.), The developing child series. London: Collins/Fontana Open Books. Trad. castellano El juego infantil (1985). Madrid: Ed. Morata.

GÖNCÜ, A. (2011). Children's pretend play as a cultural activity. Seminario de Psicología Evolutiva y de la Educación de la Universidad Autónoma de Madrid (noviembre 2011).

GönCÜ, A. y AbEl B. (2009). Activities of Children in Different Societies and Social Classes. Human Development, 52, 257-260. 
El juego es un derecho y una necesidad de la infancia

GÖNCÜ, A., Mistry, J. y Mosier, C. (2000). Cultural variations in the play of toddlers. En International Journal of Behavioral Development, 24 (31), 321-329.

Huizinga, J. (1938) Homo Ludens. Trad. castellano (2000) en Madrid: Alianza Editorial.

Karnik, R. y Tudge, J. (2010). The Reality of Pretend Play: Ethnic, Socioeconomic, and Gender Variations in Young Children's Involvement. En E. E. NwOKaH (ed.), Play as engagement and communication. Play and culture studies, vol. 10 (pp. 63-81). Lenham, MD: University Press of America.

LinAZA, J. (1992). Jugar y aprender. Madrid: Ed. Alhambra Longman.

LiNAZA, J. L. y BRUNER, J. S. (2012). La importancia del contexto cultural en el desarrollo del juego infantil. En J. A. García Madruga, R. Kohen, C. del Barrio, I. Enesco y J. Linaza (eds.), Construyendo Mentes. Ensayos en homenaje a Juan Delval. nstruyendo Mentes. Ensayos en homenaje a Juan Delval. Constructing Minds. Essays in honor of Juan Delval, cap. 13 Madrid: UNED.

LinazA, J. y Maldonado, A. (1987). Los juegos y el deporte en el desarrollo psicológico del niño. Barcelona: Anthropos.

Nelson, K. (ed.) (2006). Narratives from the Crib, Cambridge, M. A.: Ed. Harvard University Press.

Nelson, K. y Gruendel, J. (1979). Event knowledge: structure and function in development. Nueva York: Routledge.

Nelson, K. y Seidman, S. (1984). Playing with scripts. En I. Bretherton (ed.), Symbolic Play. Orlando, Florida: Academic Press. Trad. castellano en E. Turiel, I. Enesco y J. Linaza (comps.) (1989). El mundo social en la mente infantil. Madrid: Alianza Psicología.

OPIE, P. y OPIE, I. (1969). Children's Games in the street and playground, Nueva York y Oxford: Oxford University Press.

ORTEGA, R. (1991a). Un marco conceptual para la interpretación psicológica del juego infantil. Infancia y Aprendizaje, 55, 87-102.

ORTEGA, R. (1991b). El juego sociodramático y el desarrollo de la comprensión y el aprendizaje social. Infancia y Aprendizaje, 55, 103-120.

Parten, M. B. (1932). Social participation among preschool children. En Journal of Abnormal Psychology, 27, 243-269.

Pellegrini, A. D. y Smith, P. (2003). Development of Play. En J. Valsiner y K. J. Connolly (eds.), Handbook of Psychology (pp. 276-291). Londres: Sage Publications.

Piaget, J. (1932). Le Jugement morale chez l'enfant. Paris: Alcan [Trad. cast: El criterio moral en el niño. Barcelona: Fontanella, 1971].

Piaget, J. (1946). La formación del símbolo en el niño. Versión castellana, México: Ed. Fondo de Cultura Económico.

Piaget, J. (1966). Response to Sutton-Smith. Psychological Review, 73, 111-112.

Rain, N. (2011). El juego de ficción Las Visitas mapuche en el contexto sociocultural. Tesis de Diploma de Estudios Avanzados, Universidad Autónoma de Madrid.

Rogoff, B., Mistry, J., GÖnCÜ, A. y Mosier, C. (1993). Guided participatetion in cultural activity by toodlers and caregivers. En Monographs of the Society for Research in Child Development, 58, $\mathrm{n}^{\circ} 236$.

Rogoff, B. y Wertsch, J. V. (1984). Children's learning in the zone of proximal development. San Francisco: Ed. Jossey Bass.

Roopnarine, J. L., Johnson, J. E. y Hooper, F. H. (1994). Children's play in diverse cultures. New York: State University of New York Press.

Rubin, K. H., Fein, G. G. y VAndenberG, B. (1983). Play. En E. M. Hetherington (ed.), Handbook of Child Psychology: Social Development, pp. 693-774. Nueva York: Wiley.

Schwartzman, H. G. (1978). Transformations: The anthropology of children's play. Nueva York: Ed. Plenum. 
SMILANSKY, S. (1968). The effects of sociodramatic play on disadvantaged preschool children. Londres: Ed. Wiley.

Sutton-Smith, B. y Roberts, J. M. (1964). Rubrics of competitive behavior. Journal of Genetic psychology, 105, 13-37.

Sutton-Smith, B. y Roberts, J. M. (1981). Play, Games and Sports. En H. C. Triandis \& A. Herron (eds.), Handbook of Croos-Cultural Psychology, vol. 4. Boston, MA.: Allyn \& Bacon Inc.

Sylvia, K. Bruner, J. S. y Genova, P. (1976). The role of play in the problem-solving of children 3-5 years old. En J. S. Bruner, A. Jolly y K. Sylva (eds.), Play. Nueva York: Ed. Basic Books.

VyGotsky, L. S. (1978). Mind in Society: The development of higher mental processes Cambridge, M.A. Ed: Harvard University Press.

VyGotsky, L. S. (1933, 1966). El papel del juego en el desarrollo. En L.S. VyGotsky, Mind in Society: The development of higher mental processes. Cambridge, M. A. Ed: Harvard University Press. Versión castellana, Crítica: Barcelona (1982). También en http://www.marxists.org/archive/vygotsky/ works/1933/play.htm

\begin{abstract}
Play is for children both a need and a right

In the Introduction the author discusses the universal character of children's play and its relationship with local cultural practices. This is done with reference to two main contemporaries theories about play development: those of Piaget and Vygostsky. It is followed by a description of different types of children's play and it is pointed out the relevance that pretend play (make believe play) had in intervention studies conducted in the second half of the XX century. The relationship between culture and play, both in games with rules as in pretend play, shows the relevance of culture to understand and interpret children's play activities. The article concludes with an analysis of Bruner's on the relationship among education, epistemology and play. He urges not to reduce education to the transmission of knowledge as content, but to stimulate children to explore the process of knowing and understanding, to initiate them as epistemologists. Their attitudes while playing should reassure us that this is possible.
\end{abstract}

Key words: Play, Development, Culture, Intervention, Epistemology.

\title{
Résumé
}

\section{Le jeu est un droit et une nécessité pour les enfants}

Dans l'introduction de cet article, l'auteur examine le caractère universel du jeu chez les enfants et sa relation avec les pratiques culturelles locales. Il le fait en référence aux deux principales théories contemporaines sur le développement psychologique des enfants: celle de Piaget et celle de Vigotsky. Il décrit ensuite divers types de jeux d'enfants et fait remarquer la pertinence des jeux de fiction des enfants dans les études d'intervention faites dans la seconde partie du $20^{\mathrm{e}}$ siècle. La relation entre la culture et le jeu, autant dans les jeux organisés que dans les jeux de fiction, démontre la pertinence de la culture pour comprendre et interpréter les activités ludiques des enfants. L'article se termine par une analyse de Bruner sur la relation entre l'éducation, l'épistémologie et le jeu.

116 • Bordón 65 (I), 2013, 103-/I7, 155N: 0210-5934 
L'auteur exhorte à ne pas réduire l'éducation à une transmission du contenu du savoir, mais plutôt à stimuler les enfants à explorer le processus de la connaissance et de la compréhension, pour les initier comme des épistémologistes. Leurs attitudes au jeu devraient nous convaincre de cette possibilité.

Mots clés: Jeu, Développement, Culture, Intervention, Épistémologie.

\section{Perfil profesional del autor}

\section{José Luis Linaza}

Licenciado en Filosofía y Letras por la Universidad Complutense de Madrid y doctor en Psicología por la Universidad de Oxford. Catedrático de Psicología Evolutiva y de la Educación en la Universidad Autónoma de Madrid, de la que fue vicerrector. Profesor visitante en la Universidad de California y en la Universidad de Harvard. Entre sus principales líneas de investigación se encuentra el estudio de la interacción adulto-niño, los deportes y juegos en el desarrollo infantil, el juego en diferentes culturas y los procesos de socialización, marginación y exclusión social. Es miembro del Observatorio del Juego infantil y presidente de la Fundación Educación y Desarrollo.

Correo electrónico de contacto: joseluis.linaza@uam.es 